\title{
Análisis crítico de la producción de la división social del espacio residencial para el caso del Área Metropolitana de San Salvador, El Salvador, entre 1950 y 1979. Una interpretación geográfica
}

\author{
Critical analysis of the creation of the social division of \\ residential space for the case of the Metropolitan Area of San \\ Salvador, El Salvador from 1950 to 1979. A geographical \\ interpretation.
}

\author{
Carlos Vinicio Coreas-Bonilla ${ }^{l}$ \\ Universidad Nacional Autónoma de México, México
}

\begin{abstract}
Resumen
Se analiza la producción de desigualdad socioespacial en el Área Metropolitana de San Salvador, El Salvador, entre 1950 y 1979, a partir de la crítica y utilización de la noción de división social del espacio residencial elaborada por Emilio Duhau, fortaleciendo este concepto a partir de herramientas teóricas de la geografía radical y el análisis sociológico de clases, dando lugar al marco teóricoconceptual propuesto. A partir de esta formulación se dialoga con una selección de estudios que tratan sobre el proceso de urbanización, la problemática de la vivienda o las políticas de vivienda en la metrópoli salvadoreña que aportan información sobre el proceso de ocupación habitacional de las diversas clases sociales su interior, incorporando, además, otros estudios que brindan el contexto económico, político y social del periodo analizado. El estudio evidencia los efectos del modo de producción capitalista sobre la división social del espacio residencial enfatizando el papel de las políticas de vivienda y de los procesos de ocupación del suelo de las clases populares.
\end{abstract}

1 Candidato a Doctor en Geografía por la Universidad Nacional Autónoma de México (UNAM); Maestro en Población y Desarrollo por FLACSO, México; Licenciado en Sociología por la Universidad de El Salvador. Dirección de correspondencia: Avenida 4, Block 2, Casa 46, Residencial San Luis, San Salvador, El Salvador. E-Mail: vinicio.coreas@gmail.com,vinicio.coreas@comunidad.unam.mx 
Palabras clave: División social del espacio residencial, producción del espacio, desigualdad, vivienda, clases sociales

\begin{abstract}
The creation of socio-spatial inequality in the Metropolitan Area of San Salvador, El Salvador, from 1950 to 1979 , is analyzed from the critique and use of the notion of social division of residential space elaborated by Emilio Duhau, strengthening this concept from theoretical tools of radical geography and sociological analysis of classes, giving rise to the proposed theoretical-conceptual framework. Starting from this formulation a discussion is initiated through a selection of studies dealing with the urbanization process, the housing problem or housing policies in the salvadorian metropolis that provide information on the process of housing occupancy of various social classes. Other studies are also incorporated which provide the economic, political and social context of the analyzed period. The present study exhibits the effects of the capitalist mode of production on the social division of residential space, emphasizing the role of housing policies and land occupation processes of the popular classes.
\end{abstract}

Keywords: Residential space; Social division; Space creation; Inequality; Housing; Social classes.

\title{
Introducción
}

Que unas personas posean grandes y lujosas mansiones [...] mientas una gran mayoría vive en ranchos de paja, mesones o champas de cartón, está relacionado entre sí [...] Nuestra sociedad está estructurada de tal forma que los [..] recursos de que dispone, en todos los órdenes de la vida social, se distribuyen de forma jerarquizada, privilegiando a un grupo minoritario [...]. Es una estructura perfectamente tramada, que favorece a unos pocos a costa de la mayoría; que extrae los recursos económicos de esa mayoría [...], para entregársela en su mayor parte a los más privilegiados (Montes, 1979, p. 89-90).

El presente artículo se enmarca en un proyecto más extenso de investigación doctoral que trata sobre el papel que han jugado algunas instituciones estatales en la producción de desigualdad socioespacial en el Área Metropolitana de San Salvador (AMSS), a partir de políticas de vivienda implementadas desde 1989, año en que inicia el proceso de ajuste estructural y reestructuración económica. Para ese año ya existía un patrón espacial identificable que localizaba a las clases sociales con menores ingresos hacia el norte y el oriente capitalino y metropolitano, mientras que los grupos sociales privilegiados se concentraban mayoritariamente hacia el surponiente. En este sentido, surgió la necesidad de analizar las causas que dieron origen a esta desigual distribución espacial de clases en un capítulo 
de antecedentes. Este artículo forma parte de dicho capítulo y se ubica entre los años 1950 y 1979, periodo histórico de urbanización acelerada, de creación de instituciones estatales orientadas hacia la construcción de viviendas urbanas y del surgimiento de asentamientos populares urbanos; procesos que en conjunto formaron el patrón de división social del espacio residencial en el AMSS, que aún persiste. Sin embargo, si bien este concepto resulta útil para describir la estructura desigual del espacio metropolitano en función de la localización de los diversos grupos sociales, muestra limitaciones para explicar las razones de fondo dicha condición. Es por ello, que se recurre a la noción general de producción del espacio, para identificar la articulación de algunos mecanismos subyacentes que engendran la división social del espacio residencial y con ello aportar una interpretación científica y geográfica sobre la realidad salvadoreña en un periodo histórico específico (1950 - 1979) y en un área geográfica delimitada (el AMSS), que explica en gran medida la persistencia de desigualdad socioespacial metropolitana actual.

En la primera parte se presenta brevemente el área de estudio en el que se inscribe el artículo y las características generales del AMSS durante el periodo analizado. La segunda parte se conforma por el marco teóricoconceptual y el marco metodológico. En el marco teórico-conceptual se abordan algunos planteamientos generales sobre lo que se entiende por espacio geográfico y sobre la producción de este. Seguidamente, se presenta la definición general del concepto de división social del espacio residencial propuesta por Emilio Duhau (2003, 2013), para después abordar, críticamente, esta definición y establecer algunas precisiones teóricas que le darán un sustento analítico más robusto a dicho concepto, desde la noción de la producción social del espacio y del análisis de clase. En el marco metodológico se exponen los criterios de selección de estudios asociados a esta problemática y de su diálogo con el marco teórico-conceptual. Finalmente, en la tercera parte se presenta la discusión entre la propuesta teórica y la información contenida en dichos trabajos, reinterpretando la realidad salvadoreña desde la geografía crítica y brindando nuevas herramientas de análisis sobre la producción de división social del espacio residencial metropolitano. 


\section{Área de estudio}

El presente caso de estudio se enmarca en los estudios urbanos, donde el "espacio urbano hace referencia a la ciudad en tanto fenómeno histórico-social-espacial" (Soja, 2008 . p. 36) desde una perspectiva radi$\mathrm{cal}^{2}$. Asimismo, retomando a Lefebvre (1976), el espacio urbano es una forma de encuentro y reunión simultánea de los elementos contradictorios que constituyen la vida social en su totalidad en el marco de las relaciones sociales de producción clasistas, mismas que le dan contenido. Este espacio urbano estudiado está conformado por el Área Metropolitana de San Salvador, en El Salvador. Se presta mayor interés a lo que Hall (2001) denomina, como la preocupación explicativa de la geografía urbana respecto a los patrones y procesos de la estructura urbana, en el marco de una aproximación moderna estructuralista, en donde se establece que las relaciones sociales y espaciales se determinan o son influenciadas por el modo de producción capitalista. En este sentido, se analiza el proceso de urbanización acelerada del Área Metropolitana de San Salvador a partir de los modelos de acumulación capitalista, fundamentalmente del proceso de industrialización, aunque considerando también los efectos del modelo de acumulación agroexportador.

\section{Características generales}

Entre los años 1950 y 1979, en el vértice sur-poniente urbano del Área Metropolitana de San Salvador, conformado por los tres municipios de Nueva San Salvador, Antiguo Cuscatlán y la zona sur-occidental de San Salvador, se asentaron mayoritariamente clases sociales económicamente solventes, con ingresos intermedios o altos. En el resto de municipios se asentaron clases sociales de ingresos intermedios y algunas de ingresos altos, pero también las mayores proporciones de clases populares y de bajos ingresos, en la diversidad de tipologías de ocupación y poblamientos que dieron origen al problema de la vivienda: tugurios, colonias ilegales y campamentos, que se sumaron a los mesones originados a principios del siglo XX, produciendo el patrón general de la división social del espacio residencial en la metrópoli (Figura 1). Lo anterior, se desarrolló en el marco de la implementación de

2 Se retoma la geografía radical en tanto las argumentaciones elaboradas para este estudio responden a los aportes que el marxismo brinda a la geografía; y que forma parte del conjunto de abordajes críticos de la disciplina geográfica. 
modelos de acumulación económicos - mediados por un Estado militar modernizante y que buscó su legitimidad- que afectaron sobre el crecimiento poblacional, la ocupación y el poblamiento metropolitano.

\section{Marco teórico-conceptual: la producción social del espacio}

La noción de espacio adoptada es la de espacio geográfico que "podemos entender en su sentido más general como el espacio de la actividad humana" (Smith, 2006, p. 59). Asimismo, el espacio geográfico es "la totalidad de relaciones espaciales organizadas [...] en patrones identificables, [... que] son expresión de la estructura y el desarrollo del modo de producción [... siendo] más que la simple suma de las relaciones separadas que comprenden sus partes" (p. 82). Esta noción de totalidad se complementa con la propuesta de David Harvey (1977), para quien el espacio es relacional: "algo contenido en los objetos [...] un objeto existe sólo en la medida en que contiene en su interior y representa relaciones con otros objetos" ( $p$. 6), y lo ejemplifica a partir del urbanismo: "el centro urbano [...] es considerado como algo que 'contiene' una periferia, dado que no puede existir un centro sin periferia y de ese modo cada factor ayuda a definir el otro" (p. 9). La noción relacional ${ }^{3}$ implica una relación dialéctica, en la que el contenido de determinado espacio, su esencia y sentido, se encuentra relacionado y condicionado por el resto de los espacios existentes producidos. Como es obvio a partir del ejemplo de Harvey (1977), el carácter relacional del espacio geográfico también se aplica al espacio urbano. Posteriormente, Harvey (1994) argumenta sobre el sentido relacional del espacio en tanto éste no tiene existencia independiente de los diversos procesos sociales, cada uno de los cuales crea múltiples y heterogéneos espacios específicos, siendo uno de ellos el dominante y que refleja los intereses de los poderes dominantes.

Por otra parte, el espacio geográfico urbano es socialmente producido y, siguiendo a Lefebvre (1976), su producción supone el uso de fuerzas productivas y técnicas, así como la iniciativa de individuos, grupos o clases sociales con capacidad de intervención a gran escala, que a la vez son portadores de ideologías y de representaciones espaciales que se

3 Otros autores fuera de la disciplina geográfica también advierten el carácter relacional de la producción social del espacio: Saraví (2008) afirma que la desigualdad socioespacial es un proceso esencialmente relacional; mientras que Segura (2014), retoma de Burchardt (2012), que la ciudad es una realidad espacial y relacional que influye y condiciona las desigualdades sociales. 
Carlos Vinicio Coreas-Bonilla

Critical analysis of the creation of the social division of residential space for the case of the Metropolitan Area of San Salvador, El Salvador from 1950 to 1979. A geographical interpretation.

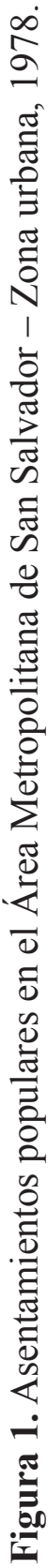

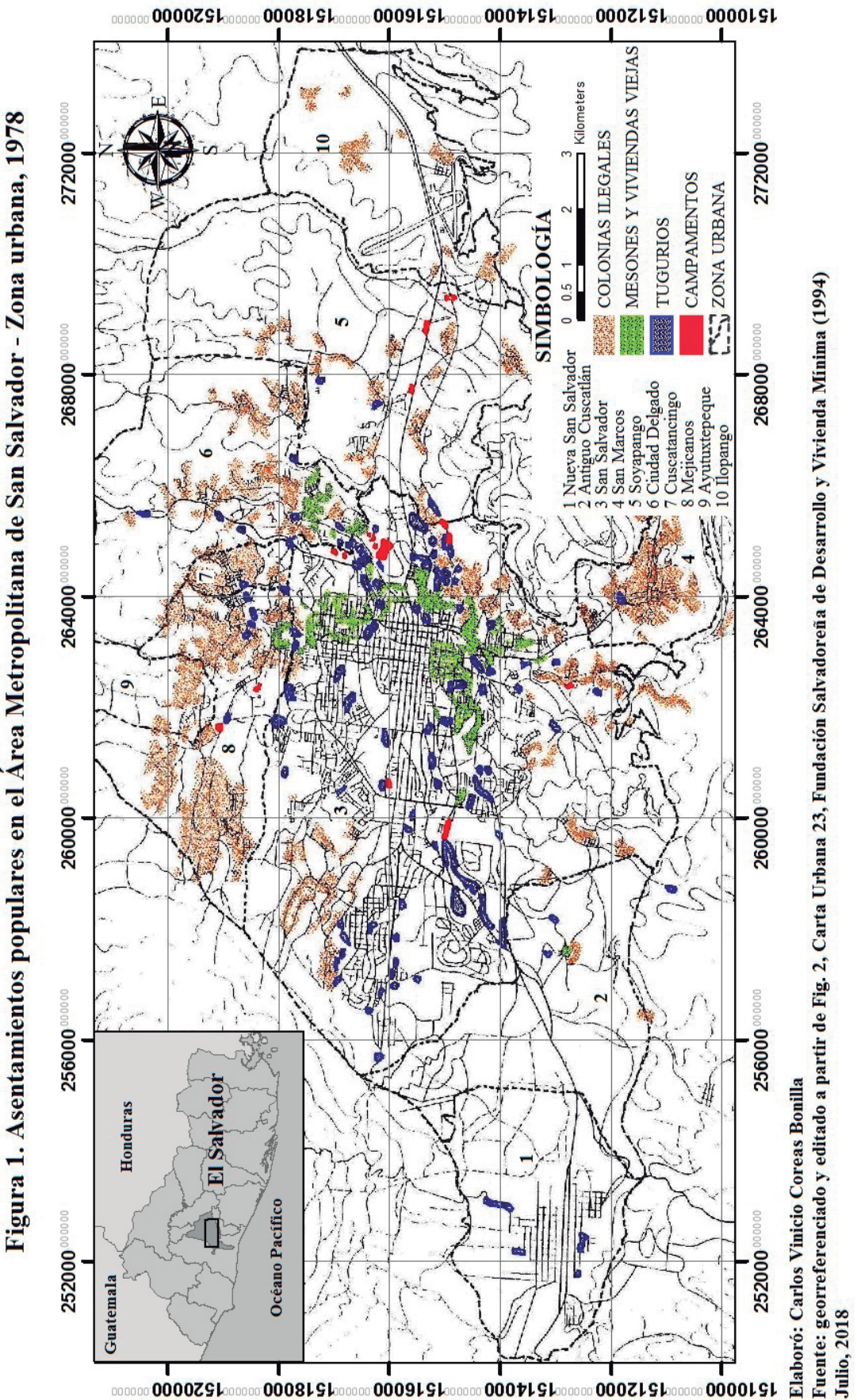


corresponden con dichas clases y grupos sociales en el marco de las relaciones de producción. Sobre las representaciones espaciales, Lefebvre (2013) advierte que éstas se vinculan con el orden impuesto por las relaciones de producción, en tanto son el espacio concebido por científicos, urbanistas, planificadores, entre otros, que dan como resultante el espacio dominante en las diversas sociedades. Con ello, el autor enfatiza que serán ciertos grupos dominantes en la sociedad quienes orientan las fuerzas productivas, a la vez que orientan la resultante general de la producción social del espacio. Otro concepto adicional propuesto por el autor ${ }^{4}$ es el de las prácticas espaciales, que hacen referencia a la producción y reproducción de "lugares específicos y conjuntos espaciales propios de cada formación social" (p. 92), es decir, a la materialización del espacio. Ambos conceptos son de gran importancia en tanto se encuentran directamente vinculados con la producción del espacio en su manifestación material.

\section{La división social del espacio residencial}

La noción sobre división social del espacio que propone Duhau (2003) resulta útil porque permite acercarnos en un primer momento a la identificación de las desiguales condiciones de ocupación del suelo con fines habitacionales -y no únicamente como un criterio de "localización"en la escala urbana o metropolitana por parte de las diferentes clases sociales. De acuerdo con Duhau (2003), la división social del espacio hace referencia a "las diferencias existentes en la localización intraurbana o intrametropolitana de diferentes grupos, estratos o clases sociales, relacionadas fundamentalmente con el mercado inmobiliario" (p. 177). En este sentido, es pertinente considerar que Duhau (2003) toma distancia de una "noción genérica" del concepto de división social del espacio que puede utilizarse como sinónimo de segregación urbana, residencial o social al introducir de forma explícita la vinculación entre localización urbana o metropolitana, clases o grupos sociales y mercado inmobiliario; aspecto que no es parte fundamental de otras formas para referirse a diferentes tipos de organización del espacio urbano o metropolitano o de desigualdad

4 Lefebvre (2013) también introduce el concepto de espacios de representación, que se refieren al "espacio vivido a través de las imágenes y los símbolos" (p. 98) de quienes habitan el espacio. Debido a lo reducido de estas páginas y a que no corresponde al interés principal de este trabajo, los espacios de representación no serán examinados. 
socioespacial ${ }^{5}$. Asimismo, Duhau (2003) agrega que lo relevante es entender cómo se expresa espacialmente, cómo se explica su dinámica y qué efectos tiene sobre otros fenómenos sociales y qué tan dividido está el espacio. La explicación que da sobre las causas de la división social del espacio las enmarca en la producción del espacio urbano, acotando que esta es el resultado de un proceso de largo plazo en el que influye una diversidad de factores, pero que fundamentalmente responde "a la lógica del mercado de vivienda y a las formas de producción y transformación/reproducción del espacio urbanizado, relacionadas con dicho mercado [...] que incorpora $[\ldots]$ la distribución de bienes y equipamientos públicos” (p. 180-181); asimismo, considera que son los ingresos ${ }^{6}$, relativamente homogéneos o similares entre individuos y/o grupos, los que definirían a los diferentes estratos de grupos o clases sociales distribuidos en el espacio. En un trabajo posterior, Duhau (2013) utiliza la expresión división social del espacio residencial, para enfatizar y hacer explícito el carácter particular de la división social del espacio a la que se refirió desde su trabajo en 2001, y agrega que este fenómeno se refiere a las "formas espaciales que adopta la distribución residencial intraurbana o intrametropolitana de los distintos estratos socioeconómicos [...] de una aglomeración urbana" (p. 81); denominando a su vez "estructura socioespacial al conjunto de las formas espaciales discernibles" (p. 81-82) que adopta la división social del espacio residencial. Duhau (2013) advierte que la división social del espacio residencial es resultado de la producción del espacio residencial, urbano o metropolitano que determina "a través del funcionamiento del

5 Por ejemplo, la noción de segregación urbana de Castells (1976), se refiere al caso o los casos "en que la distancia social tiene una fuerte expresión espacial", de tal forma que la segregación urbana es "la tendencia a la organización del espacio en zonas de fuerte homogeneidad social interna y fuerte disparidad social entre ellas" (p. 204). Pero esta definición no necesariamente hace referencia de forma explícita al mercado inmobiliario y a su relación con las diversas clases sociales, sino, a lo sumo, a "los lugares de residencia", entendidos como una referencia de localización más que respecto al mercado inmobiliario. Como ejemplo de esta noción de lugar de residencia y no del mercado inmobiliario respecto al concepto de segregación, se puede retomar, entre muchos, la planteada por Ariza y Solís (2009), quienes analizan la "segregación residencial socioeconómica" para el caso mexicano sin considerar aspectos vinculados al mercado inmobiliario, centrándose en la conformación de grupos sociales a partir de variables como los ingresos laborales, la ocupación, o el nivel de escolaridad. El mismo Duhau (2003) diferencia el concepto de división social del espacio respecto a otras formas de entender la segregación "fuerte", como es el ejemplo del apartheid sudafricano o los guetos judíos de Varsovia. Aunque reconoce que algunas de estas nociones alrededor de la desigualdad socioespacial pueden ser "cercanas", esto no significa que sean iguales o sinónimos de la división social del espacio según su propuesta.

6 Duhau (2003) reconoce que podría haber otras variables o características sociales que pudieran incorporarse en el estudio de la división social del espacio, pero el énfasis está en el mercado inmobiliario. 
mercado inmobiliario, el tipo de vivienda y las áreas en las que esta estará localizada, de acuerdo con el nivel socioeconómico de los hogares" (p. 82); y agrega que las políticas públicas también pueden contribuir a la división social del espacio residencial, pero no inscribe la noción de producción del espacio residencial en las relaciones sociales clasistas del modo de producción capitalista, con todas las consecuencias en el análisis que esta omisión podría significar.

\section{La producción de la división social del espacio residencial}

Como se apuntó, la noción de división social del espacio y de división social del espacio residencial que desarrolla Duhau $(2003,2013)$ es útil en un primer momento para establecer de forma explícita la articulación entre mercado de inmobiliario (con énfasis explícito en el mercado de vivienda), clases sociales y localización urbana o metropolitana. Sin embargo, se propone avanzar hacia un acercamiento que devele y haga énfasis en los mecanismos y relaciones sociales que están ocultas en el modo de producción capitalista y que son la causa última de este fenómeno socioespacial. En lo subsecuente, se lleva a cabo una revisión crítica respecto a la noción de la división social del espacio residencial de Duhau (2013) (con el énfasis explícito de lo "residencial" asociado al mercado inmobiliario que ya se vislumbraba desde 2003), para aportar seis precisiones que abonan a un acercamiento de mayor profundidad sobre sus causas. La primera consiste en interpretar la división social del espacio residencial como resultado de las prácticas espaciales a las que se refiere Lefebvre (2013), es decir, la producción material y física a partir de la reproducción de las relaciones sociales de producción en el capitalismo, en la que intervienen representaciones espaciales que lo han preconcebido, al menos parcialmente. La segunda precisión consiste considerar que la estructura socioespacial que adopta la división social del espacio residencial (Duhau, 2013), son los patrones espaciales identificables que resultan de la estructura y el desarrollo del modo de producción capitalista a los que se refiere Smith (2006).

La tercera precisión es de mayor relevancia, en tanto critica la causalidad del mercado en la producción de la división social de espacio. Smith (2006) indica que "la totalidad de las relaciones espaciales organizadas [... son] expresión de la relación entre capital y trabajo" (p. 82), es decir, de 
la relación entre las clases y los grupos sociales que se expresan de forma fenomenológica en el mercado. En este sentido, conviene retomar el planteamiento de Marx (1995) respecto a las relaciones mercantiles, es decir, de oferta y demanda:

lo que regula el principio de la demanda, se halla esencialmente condicionado por la relación de las distintas clases entre sí por su respectiva posición económica $[\ldots]$ nada absolutamente puede explicarse por la relación entre la oferta y la demanda si no se expone previamente la base sobre la que descansa esta relación (Marx, 1995, p. 185-186).

Así, la causalidad de la división social del espacio residencial se encuentra en las relaciones de clase que son la base de la relación entre oferta y demanda de vivienda: es la posición de clase la que define los ingresos que permiten enfrentar a las personas al mercado. Según Wright (2010), las personas se relacionan unas con otras a partir de los modos de producción y son estas relaciones, en última instancia, las que determinan el ingreso $^{7}$. Adicionalmente, Emilio Pradilla (1984) observa que la distribución de lo socialmente producido "asume la mediación entre producción y el consumo", "reparte según las leyes sociales" determinando la proporción, el cuánto de los productos le corresponden a cada individuo a partir del intercambio que "aporta los productos particulares por los que [... se ] desea cambiar la cuota que le ha correspondido [al individuo] a través de la distribución" (p. 242-243). Las argumentaciones de Pradilla (1984) y las de Wright (2010) implican que las relaciones de clase y de distribución social, en el marco del modo de producción capitalista, están detrás del ingreso y del acceso a la vivienda como valor de cambio o valor de uso.

La cuarta precisión aborda el carácter relacional, tanto de las clases sociales como del espacio, por sobre una concepción de "estratificación". Crompton (2013) señala la distinción teórica que existe entre construir las clases a partir de estratos ordenados, según ciertas características o atributos, por ejemplo, los ingresos, en las que no se evidencia ninguna relación

7 Diferentes estudios relacionales (sociológicos) de clase dan cuenta de que estas son las que determinan los ingresos. Solís y Benza (2013) suscriben que las clases sociales implican brechas de ingresos en sus investigaciones sobre algunos países de Latinoamérica. Asimismo, Coreas (2014) comprobó empíricamente para el caso particular de El Salvador que la clase social condiciona los ingresos y la propensión a la pobreza. Este resultado lleva a plantear que la clase social, entendida relacionalmente, condicionaría el acceso a la vivienda en el mercado. 
social $^{8}$; respecto a su abordaje desde una noción relacional, que da cuenta de un sistema de relaciones sociales, fundamentalmente asociado a las relaciones laborales en las que las clases sociales condicionan mutuamente su existencia. Rafael Feito (2007) reitera que "las concepciones relacionales definen las clases por su relación social estructurada con otras clases" (p. 31). Para las sociedades latinoamericanas, Pérez Sáinz (2014) acota que en el estudio de la desigualdad social se debe dar prioridad al análisis de clase ${ }^{9}$, en tanto disputan las condiciones de generación y apropiación de excedente, que incorpora tanto la producción como al intercambio. Dicho de forma sintética, la noción relacional advierte que la existencia de clases sociales dominantes y/o privilegiadas en lo político y económico, social y cultural son interdependientes y condicionantes de la existencia de clases subordinadas en lo político, económico, social y cultural. El carácter relacional de clase se impone conceptual y teóricamente a la noción de estratificación social que surge de la recolección de datos, como fuente de la conformación de los grupos sociales; lo que implica un abordaje teórico y metodológico "en contra sentido" que conviene desarrollar en los estudios sobre la desigualdad socioespacial en general ${ }^{10}$.

Dentro del análisis relacional de clase, se propone incorporar al grupo social definido como "marginado", "informal" o "excluido" de las relaciones de producción capitalista. Aquiles Montoya (1995) propone que el sector socioeconómico denominado como "informal" es un grupo social que no se relaciona socialmente a partir de la compra-venta de fuerza de trabajo, es decir, por procesos de explotación directa, y que tampoco ejercen la actividad laboral bajo la lógica capitalista, sino bajo la lógica de la subsistencia que les permite su autorreproducción. El trabajo de los individuos o grupos familiares de este grupo social está subordinado y subsumido indirectamente al capital, y por esta relación se articulan con

8 Rubalcava y Schteingart (2012) advierten que en muchas ocasiones no se problematiza sobre la conformación de los diferentes estratos sociales, llevando a la utilización de términos centrales o categorías que carecen de contenido social, tales como "fragmentación, mezcla social, mayor cercanía entre grupos sociales, aislamiento, etcétera [...] que a veces pueden implicar apariencias más que relaciones sociales” (p. 18), es decir, que se quedan en el marco fenomenológico sin describir el contenido social de las relaciones que subyacen a desigualdad socioespacial.

9 El autor advierte sobre la importancia de las dinámicas de individualización y de pares categoriales que podrían llegar a relativizar las dinámicas de clase, aunque destaca su prioridad en nuestro contexto.

10 No solamente el análisis relacional de clase neomarxista desarrollado por Erik Olin Wright puede ser aplicado al estudio de la desigualdad socioespacial, sino también el análisis relacional neoweberiano desarrollado por John Goldthorpe; o bien, el abordaje de la desigualdad social relacional basado en Durkheim. 
la sociedad en conjunto y con el modo de producción capitalista, pero está subsumido en última instancia, forma parte de la totalidad social de la que surge y con la que se relaciona. Este "sector informal" -que proponemos sea denominado como clases populares- está conformado por productores de valores de uso para el autoconsumo, por productores mercantiles simples que intercambian sus productos a precios por debajo de los precios del mercado; y por comerciantes no capitalistas (generalmente minoristas). Montoya (1995) muestra teóricamente cómo la existencia de estas clases populares (los informales) contribuyen a la valorización extraordinaria del capital, es decir, son funcionales y se articulan a los procesos de acumulación capitalista.

En cuanto al carácter relacional de la división social del espacio residencial, retomando a Harvey $(1977,1984)$, la existencia de zonas o espacios donde prevalecen y habitan las clases dominantes y/o privilegiadas que realizan el valor de uso de viviendas, cuyas cualidades están "hechas a su medida", no tendría sentido ni contenido sin la existencia de otras zonas o espacios donde viven las clases populares, socialmente deprimidas o de menores ingresos con valores de uso de igual modo "hechas a su medida". La totalidad social y espacial heterogénea es coherente y dominada por y con el modo de producción capitalista y sus relaciones sociales inherentes. En este sentido, Lefebvre (1976) explicita que el contenido que subyace en la forma del espacio urbano asociada a la desigualdad socioespacial y a la existencia de espacios periféricos y pobres permite "la reproducción de las relaciones de producción que son relaciones de clase" (p. 70).

La quinta precisión es sobre los límites de la noción del mercado cuando se aplica a la vivienda y al suelo, en tanto ambos representan un valor de uso realizado o potencial, pero no siempre implican un valor de cambio, aunque potencialmente puede llegar a serlo. Esto sucede en las ocasiones de ocupación de hecho del suelo urbano y autoproducción (autoconstrucción) de viviendas, que en la mayoría de las veces pueden localizarse en suelo estatal, pero de igual manera puede ocurrir en suelo privado $^{11}$. Algunas de las principales urbes latinoamericanas han sido producidas socialmente en una medida no despreciable a partir de estos

11 Si bien Duhau (2013) advierte la existencia de un mercado formal y no formal, la relación de mercado siempre está presente, dejando de lado aquellos casos en los que no hay relación de compra-venta alguna, a pesar de que en trabajos anteriores, como en Hábitat popular y política urbana de 1998 haya reconocido este tipo de ocupación de suelo urbano por parte de las clases populares. 
procesos al margen del mercado, dando lugar a patrones espaciales identificables (Smith, 2006) que forman parte de la división social del espacio residencial producido por las clases populares, en directa relación con el modo de producción capitalista.

La sexta y última precisión tiene que ver con las fuerzas productivas (medios de producción y fuerza de trabajo) y las técnicas que intervienen en la producción del espacio, tal como lo afirma Lefebvre (1976). Milton Santos (1990) interpreta el espacio humano (o espacio geográfico) como resultado de la producción, donde el acto de producir es un acto de producir espacio, siendo las técnicas y los instrumentos de trabajo mediadores de la relación humana con la naturaleza. Las técnicas son "un conjunto de medios instrumentales y sociales, con los cuales el hombre realiza su vida, produce y al mismo tiempo, crea espacio" (p. 27). Para Santos (2000) existen diferentes técnicas, según diversos grupos sociales, que al mediar con el territorio dan como resultado espacios diferenciados, lo que implica la asociación de ciertas técnicas a grupos determinados que producen desigualdad socioespacial. La técnica se convierte en mediadora de la producción división social del espacio residencial.

Incorporando los argumentos referidos a las técnicas con las formas de producción de viviendas expuestas por Emilio Pradilla (1987) se evidencia su desigual distribución y apropiación entre las diferentes clases sociales. En el proceso de autoconstrucción de vivienda el productor de esta es también quien realiza el valor de uso, caracterizándose este producto por sus condiciones de precariedad, agravado por la situación de quedar aislada de servicios básicos en muchos casos. Retomando estos planteamientos, serían sobre todo las clases populares las usuarias de técnicas e instrumentos de trabajo más rudimentarios en el proceso de autoproducción de sus viviendas. En algunos casos, la producción de estas viviendas podría suponer costos de localización, siempre y cuando se efectúen sobre suelo privado y que el propietario del mismo venda o arriende el terreno sobre el que se edifica la vivienda, en cuyo caso la vivienda entraría en el marco del mercado informal de la vivienda y del suelo; o bien, puede no representar renta alguna si las viviendas son producidas por medio de procesos de ocupación de suelo estatal, en la que la vivienda escapa -por lo menos temporalmente- de relaciones mercantiles. 
En la producción manufacturera, Pradilla (1987) advierte que esta se impulsa y lleva a cabo en el marco de la relación de quienes realizarán el valor de uso de la vivienda producida y las empresas constructoras que venden sus servicios constructivos a la demanda solvente perteneciente a clases sociales, cuyos ingresos pueden ser medios o altos. Quienes entran en relación con este tipo de empresas son personas o grupos familiares propietarias del suelo donde se lleva a cabo el proceso productivo. En este caso, el proceso productivo sería desarrollado de forma general por una sola empresa que debería contar con una cantidad limitada pero suficiente de fuerza de trabajo, recursos tecnológicos e instrumentos de trabajo para poder construirla, dando como resultado una vivienda de buena calidad. La vivienda producida bajo estas condiciones puede presentar de forma inmediata su valor de uso, o bien, estar destinada a ser un valor de cambio.

Finalmente, el proceso de producción industrial de vivienda se caracteriza, según Pradilla (1987), por la desaparición de la relación directa entre quien realiza el valor de uso de la vivienda y los múltiples agentes -públicos y/o privados- que intervienen en la construcción de vivienda, bajo la forma de mercancía acabada. Los agentes que intervienen entre la producción y el intercambio van desde los propietarios del suelo, empresas constructoras, agentes inmobiliarios, agentes financieros de capital estatal o privado, entre otros. De forma general, la mercancía-vivienda es producida en serie, lo que requiere una intervención y mediación técnica mucho más sofisticada y eficiente. Este tipo de producción permite mayores márgenes de ganancia o ganancias extraordinarias respecto a la producción manufacturera. En general, los materiales para este tipo de producción de vivienda son de buena calidad.

Cada una de las formas que asume el proceso productivo de las viviendas (Pradilla, 1987) estaría asociada a desiguales propiedades espaciales, definidas como "la forma en que el valor de uso se presenta-su extensión espacial [...] y su forma resultante" (Smith, 2006, p. 81-82), a lo que añadimos las características de los materiales con los que se ha producido el valor de uso. Además, el resultado del espacio reproducido en cuanto a sus propiedades "no es [...] un dato natural o abstracto, sino una construcción social que se caracteriza por sus rasgos físicos, por la propiedad socialmente determinada de recursos (o carencia de ellos) y por su organización simbólica" (Rapport, 1977; en Martín-Baró, 1985, p. 39), es decir, no es neutral, natural 
o preestablecido; lo que a su vez se relaciona con la mediación técnica en su producción (Lefebvre, 1976; Santos, 1990, 2000); y finalmente, dan cuenta de patrones espaciales identificables que son expresión de la estructura y el desarrollo del modo de producción capitalista (Smith, 2006).

\section{Marco metodológico}

El abordaje es cualitativo y se sustenta en los postulados teóricos, desarrollados en el marco teórico-conceptual, que parten del prisma de la geografía urbana radical que incorpora el análisis de clase ${ }^{12}$. Se establece un diálogo entre esta propuesta teórica y un conjunto de estudios que abordan el proceso de urbanización, la problemática de vivienda o las políticas de vivienda del AMSS entre 1950 y 1979. Se parte de una discusión que va de lo general en lo político, económico y social hacia lo particular de la división social del espacio residencial, en una comunicación entre lo teórico y lo empírico, planteado en los estudios retomados, mismos que presentan aportes sustantivos de información sobre el proceso de ocupación y localización de las diversas clases sociales en la metrópoli, así como del contexto económico, político y social. En este sentido, la selección de estos se sustenta en criterios de fondo en cuanto a su contenido, a partir de la experiencia desarrollada en el proceso de investigación y comprensión de la realidad salvadoreña; lo que no implica que los trabajos no considerados carecen de relevancia, pero se afirma, que básicamente reforzarían las aportaciones de los estudios efectivamente incorporados. La escala de observación que se retoma en este trabajo, hace referencia a "los grandes lineamientos de la organización del espacio urbano", que se alinean con el concepto de división social del espacio residencial, que es diferente de la segregación en tanto esta última alude a una escala de observación "más

12 La intención del análisis de clase no debe interpretarse en ningún momento como un abordaje reduccionista que elimina las posibilidades de agencia. Basado en un enfoque sociológico relacional de clase, John Goldthorpe (2010) advierte que las acciones de los individuos y grupos sociales "están condicionadas por la distribución de los recursos y, a su vez, de las oportunidades y constricciones implicadas en el conjunto de la estructura de clases"( p. 286); y que "los diferenciales [sociales] persistentes son simplemente una expresión del modo en que las distribuciones desiguales de oportunidades y constricciones características de una sociedad de clases contribuyen a su propia perpetuación" (p. 301). En este sentido, la agencia individual o colectiva están supeditadas a la posición de clase, es decir, a márgenes sociales para la elección y la acción; y no dentro del marco de una absoluta e irrestricta libertad de agencia. 
desagregada", es decir, a una mayor escala de observación ${ }^{13}$ (Rubalcava y Schteingart, 2012).

\section{Resultados y discusión sobre la producción de la división social del espacio residencial en el AMSS, 1950-1979}

El periodo histórico comprendido entre 1950 y 1979 en El Salvador puede ser considerado, como proponen Griffith y González (1999), como un periodo de "consolidación de un Estado con un proyecto de nacionalización (industrializante)" con autonomía intermedia entre el Estado y las clases dominantes nacionales, en el que la sucesión de gobiernos y sus instituciones, conducidas principalmente por militares de carrera, "justifica el papel interventor del Estado en los ámbitos económico y social” (p. 898). Esta autonomía se logró legitimando el papel de los militares en el gobierno a partir de su función como mediadores entre tres grandes intereses: a) los del sector oligárquico tradicional, cuyo eje de acumulación se sustentaba en la producción agroexportadora; b) los de otras fracciones de la oligarquía que, en alianza económica con industriales estadounidenses, impulsó el proyecto de industrialización; y c) los de ciertos sectores de la clase trabajadora dentro del sector público y el privado, así como de algunos sectores de las clases populares urbanas que crecieron con intensidad durante estas décadas.

El apoyo a la oligarquía tradicional se dio a partir de la producción de infraestructura funcional a este modelo de acumulación en la escala nacional. En este sentido, Lungo (1996) acota que se invirtió en infraestructura vial y comunicaciones para la expansión agrícola orientada hacia la exportación. Por otra parte, el apoyo al proceso de industrialización se llevó a cabo por medio de la producción de infraestructura urbana y de generación de energía eléctrica (López Bernal, 2015). Según López Pérez (1986), sólo en la zona metropolitana de San Salvador las inversiones gubernamentales durante la década de 1960 fueron equivalentes al 55\% de la inversión en infraestructura urbana a nivel nacional. Asimismo, el gobierno militar y el resto de las instituciones de los poderes formales del Estado también

13 En este sentido, la "escala de observación" es otra de las características que diferencia la noción de la división social del espacio residencial con respecto a otros conceptos que dan cuenta de la desigualdad socioespacial, como es el caso del concepto de segregación urbana o residencial, a pesar de que esta no haya sido una propuesta compartida por Emilio Duhau. 
lograron legitimarse con sectores de la clase trabajadora del sector privado y público ${ }^{14}$, amparados en la promulgación de la Constitución Política de 1950, cuyo contenido era socialmente proteccionista e interventor sobre el desarrollo económico y social. Esto se hizo por medio del incremento del gasto social y del bienestar público en las zonas urbanas a partir de la introducción de impuestos progresivos (Walter y Williams, 2011), lo que implicaría nuevamente el papel mediador de los militares entre las fracciones de clase oligárquicas ${ }^{15}$. A partir de la nueva Constitución se pusieron en práctica una diversidad de políticas sociales por medio de instituciones creadas en el marco de la modernización del Estado como parte esencial de su legitimación, donde las políticas de vivienda adoptan este papel y forman parte de los mecanismos de la producción de la división social del espacio residencial.

Ambos modelos de acumulación tuvieron momentos de auge y decaimiento que impactaron sobre el proceso de urbanización durante ese periodo. Por un lado, el auge en la diversificación agroexportadora y la modernización en la agricultura provocaron la expulsión y migración de la población rural hacia las ciudades (FUNDASAL, 1997a), mientras que durante el estancamiento de la estructura agraria se refuerzan los flujos migratorios, sobre todo a San Salvador (Lungo, 1980) y a sus municipios aledaños. Por su parte, el modelo de acumulación sustentado en la Industrialización por Sustitución de Importaciones promovió la configuración y concentración de un cordón industrial en los límites del municipio de Soyapango entre 1960 y 1970 que de igual manera atrajo migraciones (Barba, 1998). No obstante, este modelo de acumulación mostró sus límites por su alta dependencia de insumos importados y su reducida captación de fuerza de trabajo formal, provocando el incremento del sector servicios, pero, fundamentalmente, desde la informalidad o bajo condiciones precarias de empleo (López Pérez, 1986). Las razones del fracaso del proceso de industrialización se debe a cierta negligencia propiciada e impulsada

14 Aunque con un marcado distanciamiento de los procesos de urbanización e industrialización de países latinoamericanos como Brasil o México, el proceso de industrialización en El Salvador también generó transformaciones en la estructura de clases en el marco de un proceso de movilidad social estructural, como el que describe Filgueira (2001), en el que crecen las capas medias y la fuerza de trabajo proletarizada en el sector industrial, así como el crecimiento del poder burocrático y el aumento del sector servicios.

15 Si bien López Bernal (2015) considera que esta aplicación de impuestos se llevó a cabo "sin tener mayor resistencia por parte de los grupos de poder" (p. 41), Walter y Williams (2011) acotan que la oligarquía sí se opuso al incremento de los impuestos. 
por la oligarquía agroexportadora tradicional junto con los sectores más conservadores del poder militar (Velásquez, 2012), así como por la relocalización (o abandono) de la inversión extranjera directa de Estados Unidos en la Cuenca del Caribe (Salgado, 2016).

Los flujos migratorios dirigidos hacia las principales ciudades y sobre todo al AMSS ocasionados por los ejes de acumulación, así como los límites para la absorción de fuerza de trabajo en el sector formal dentro de la metrópoli, dieron origen en El Salvador, al problema de la vivienda urbana para las clases populares que se incorporaron a las relaciones capitalistas desde la informalidad, es decir, desde la subsunción indirecta del trabajo al capital, retomando el planteamiento de Montoya (1995). Por otra parte, como se abordará más adelante, aquellas clases sociales cuyo trabajo se inserta en el mercado laboral formal y cuyos ingresos no son los más bajos, es decir, cuyo trabajo se encuentra subsumido directamente al capital o cuyas relaciones laborales se establecen con las instituciones estatales, tendrían acceso a otros tipos de vivienda con propiedades espaciales (Smith, 2006) de mejor calidad. En este sentido, trayendo a colación a Lefebvre (2013), se puede argumentar que el espacio geográfico urbano orientado por la representación espacial y producido por la práctica espacial de poblamiento de la metrópoli, en el marco del modo de producción capitalista y los ejes de acumulación puestos en marcha, daría origen a patrones identificables de localización de las diversas clases y grupos sociales, que serían la expresión de la estructura y del modo de producción capitalista, como advierte Smith (2006); que a su vez estarían vinculados con desiguales condiciones de viviendas. Recuperando a Harvey (1977, 1994), Feito (2007), Crompton (2013), y Pérez Sáinz (2014) la problemática de la vivienda y la división social del espacio residencial producido no es independiente de los procesos sociales y económicos, existiendo una relación de interdependencia entre los espacios urbanos producidos y estos procesos, es decir, que las formas de producción de vivienda y ocupación habitacional del suelo metropolitano de las clases populares y de poblamiento del resto de clases sociales son relacionales en tanto se producen en función de su relación y posición dentro de la estructura de clases y la estructura económica (Marx, 1995).

Las formas de poblamiento de las clases populares han sido básicamente cuatro durante este periodo: mesones, tugurios, colonias ilegales y 
campamentos. De acuerdo con FUNDASAL (1994), los mesones son viejas viviendas abandonadas de las clases altas en las que sus habitaciones eran alquiladas separadamente a diferentes familias, constituyendo una opción de vivienda para sectores de bajos ingresos, que al agotarse dan origen a los tugurios y colonias ilegales. Los tugurios son asentamientos de viviendas autoconstruidas en terrenos marginales invadidos, generalmente estatales, con materiales de desecho, que no cuentan con servicios básicos del que también se valen las familias más pobres. Estos se caracterizan por su "alto grado de insalubridad, vivienda precaria, hacinamiento, promiscuidad, y tenencia de hecho del terreno". Las colonias ilegales o lotificaciones ilegales se originan en "grandes terrenos de propiedad privada ubicados en la periferia urbana [... a partir de] lotes sin servicios básicos [... para] familias de bajos ingresos que tienen que construir su propia vivienda" (p. 5); y los campamentos implican la relocalización de los damnificados por el terremoto de 1965 que, para 1975, se habían consolidado como una forma permanente de alojamiento de las clases populares. FUNDASAL (1994) destaca que los primeros tres tipos de asentamientos, característicos fundamentalmente de las clases populares, se pueden encontrar traslapados como realidad urbana.

Las definiciones de estas tipologías de asentamiento de las clases populares se enmarcan sobre todo en la autoconstrucción (Pradilla, 1987), con excepción de los mesones. Dado el carácter rudimentario de las técnicas aplicadas (Santos, 2000), los valores de uso de viviendas autoproducidas presentan propiedades espaciales (Smith, 2006) precarias, lo que implica por la misma razón, formas degradadas de autorreproducción para las personas, familias u hogares que en ellas habitan y que conforman estas clases populares. Cabe destacar el factor común de los tugurios y campamentos que se sitúan en suelo estatal -lo que implicaría estar fuera de las relaciones mercantiles- y su diferencia respecto a la colonia ilegal que se encuentra inserta en el mercado informal del suelo, sobre todo, del suelo periférico.

Los mesones, por su parte, si bien provienen de las viviendas de las clases dominantes, es decir, producidas esencialmente bajo la forma manufacturera, el paso del tiempo, la falta de mantenimiento y el reducido espacio para las familias que ocupan en las habitaciones hace que el mesón impida "una vida familiar digna" (Herrera y Martín-Baró, 1978, p. 806), lo 
que implica de igual manera condiciones degradadas para la reproducción de las clases populares que habitan en ellos.

La información sobre los asentamientos de las clases populares en el AMSS, aunque limitada y no del todo precisa, es consistente en cuanto al surgimiento, crecimiento y la importancia de los tipos de poblamiento que estas adoptaron en la metrópoli durante el periodo analizado. Para el año 1976, 8,060 (11.5\%) familias habitaban en tugurios; 4,320 (6.2\%) ocuparían campamentos; 34,130 (48.8\%) vivían en mesones; y otras $23,360(33.4 \%)$ residían en colonias ilegales, ascendiendo a un total de 69,870 (100\%) familias (FUNDASAL, 1994). Estas cifras contrastan con el total de viviendas urbanas (que podrían contener más de una familia u hogar) que, según Zschaebitz, Serarols y Portillo (1994), ascendía entre 1976 y 1977 a 149,178 unidades habitacionales. La United States Agency for International Development (1985) brinda información que establece, que entre 1976 y 1978 las viviendas del AMSS de clases las populares se agrupaban en un $23 \%$ bajo la forma de cuartos de mesón, cerca del $5 \%$ de viviendas se encontraban en tugurios y un $19 \%$ estaban en colonias ilegales, ascendiendo a un total de $47 \%$ del total de viviendas metropolitanas, lo que da cuenta del grave problema de vivienda y de reproducción de las clases populares, así como de la proporción de espacio socialmente producido, que les corresponde dentro de las leyes de distribución social (Pradilla, 1984). No obstante, el Estado intervino en algunos casos para legitimarse y legitimar las relaciones sociales clasistas que originaron el problema de la vivienda como resultado del modo de producción y los modelos de acumulación capitalista, como se señaló previamente. Esto lo hizo por medio de algunas acciones de rehabilitación de las denominadas colonias marginales a partir de la década de 1960 (FUNDASAL, 1999), y de programas de regularización de la propiedad desde la década de 1970 (FUNDASAL, 1993a).

Por otra parte, la producción social del espacio en lo que se refiere a la vivienda no ha sido exclusiva de las clases populares. Las clases sociales insertas en el mercado laboral formal contaron con dos tipos de acceso a estos valores de uso y valores de cambio: a) por medio de la intervención estatal asociada o no con el sector privado de la producción y financiamiento de vivienda; o b) por el sector privado actuando de forma unilateral. El gobierno militar buscó legitimarse con las clases y grupos 
sociales del mercado laboral formal a partir de diferentes roles alrededor de la producción, financiamiento, circulación e intercambio de vivienda, creando una diversidad de instituciones. En 1951 se funda el Instituto de Vivienda Urbana (IVU) con el objetivo de construir viviendas unifamiliares y multifamiliares (Harth, 2013). De acuerdo con Lungo (1980), la creación de este instituto se dio bajo recomendaciones norteamericanas ${ }^{16}$, que desde 1949 sugirieron la construcción y venta de viviendas para grupos sociales de bajos y medios ingresos a partir de la existencia de cuantiosos excedentes provenientes de la producción y exportación del café por parte de las clases dominantes. Durante los primeros años de existencia del IVU los fondos utilizados provenían el propio erario estatal, pero a partir de 1962 se inicia la dependencia de préstamos internacionales.

Posteriormente, Lungo (1980) advierte que en la década de 1970 se abandonan las funciones de construcción de vivienda por parte del IVU, pasando a ejercer el rol de promotor. Esta transformación del IVU ocurrió junto con la creación de la Financiera Nacional de Vivienda (FNV) en 1960 con un $51 \%$ de capital estatal y otro $49 \%$ proveniente de capital estadounidense $^{17}$. Más adelante, en 1965 se crean, bajo la regulación de la FNV, las primeras Asociaciones de Ahorro y Préstamo (AAP), como instituciones privadas financieras conformadas por accionistas capitalistas. En conjunto, el trabajo de la FNV y las AAP contribuyeron también a dinamizar la industria de producción de materiales constructivos. La FNV garantizó "al capital privado [de las AAP] su reproducción en la producción de vivienda. Sus funciones principales [...] son las de dar asistencia financiera al capital privado [...] y garantizar el pago de los deudores y la ganancia de los capitalistas" (p. 18). Las AAP, bajo la regulación de la FNV, podían fijar sus propias tasas de interés, pero manteniéndose por debajo de las de la banca comercial (FUNDASAL, 1993b). Asimismo, los organismos privados de financiamiento de vivienda, junto con empresas constructoras, se aglutinan en la Cámara Salvadoreña de la Industria de la Construcción (CASALCO) (FUNDASAL, 1993b). De acuerdo con Fontg, Barrientos y Figueroa (1991), entre 1972 y 1979 surgen siete AAP para atender a la demanda solvente y formalizada laboralmente, con créditos

16 Lungo hace referencia al documento "Recomendaciones para un programa nacional de vivienda" elaborado para la Public Administration Service.

17 Según Romero y Vides (2006), posteriormente se asociaron a las AAP algunas empresas privadas de la construcción. 
cuyas tasas de interés eran inferiores a los de la banca privada, legitimando al gobierno militarista y a la conformación general del Estado.

Otra institución estatal creada alrededor del proceso de producción y circulación de vivienda, en 1973, es el Fondo Social para la Vivienda (FSV), diseñada como institución de seguridad social que declara de "interés social" la producción de vivienda dirigida a clases sociales formalizadas. Según Lungo (1980), este fondo se orientó hacia la adjudicación de créditos para la compra, construcción, reparación o ampliación de viviendas; y sus fondos serían constituidos por un subsidio inicial del Estado durante sus primeros cinco años de funcionamiento, por la cotización patronal del $5 \%$ y de un $0.5 \%$ de los trabajadores ${ }^{18}$; a lo que se agregarían otros fondos del Estado, la ganancia o renta de su funcionamiento y otros ingresos que pudiera obtener. Adicionalmente, en 1975 se crea el Instituto Nacional de Pensiones de los Empleados Públicos (INPEP), orientado a los empleados públicos que no pertenecían a los cuerpos de seguridad y represión, para financiarles la compra de vivienda construida por el IVU, el FSV o las AAP. En lo que respecta a las fuerzas armadas, estas crearon en 1974 la Caja de Ahorro Mutual de la Fuerza Armada, que tenía dentro de sus funciones otorgar créditos hipotecarios para la compra de vivienda a los militares, y que a principios de la década de 1980 se convertiría en su instituto de previsión social (IPSFA). Otras acciones para facilitar el acceso a la vivienda por parte del Estado a las clases sociales, cuyo trabajo se encuentra directamente subsumido al capital, se ha llevado a cabo por medio de créditos desde la banca estatal, como es el caso del Banco Hipotecario de El Salvador (FUNDASAL, 1993b).

Cabe destacar que el proceso de producción de vivienda llevada a cabo por el Estado y su relación con las entidades del sector privado alrededor de este proceso productivo, de circulación e intercambio resultan ser una experiencia de importancia para la acumulación capitalista. De acuerdo con Ferrufino (2013), durante estas décadas se consolidan los factores que permiten la intervención del sector privado en la producción de vivienda masiva dirigida hacia los sectores de demanda solvente de vivienda urbana, sobre todo en su vinculación con la FNV y el FSV.

18 Esta capitalización porcentual proviene del Instituto Salvadoreño del Seguros Social (ISSS), creado en 1949. 
La producción de la vivienda a partir de la intervención estatal y su relación con el sector privado alrededor del proceso productivo requiere el uso de técnicas más sofisticadas (Santos, 1990, 2000) para la producción de la misma, que podría haberse realizado a partir de la forma manufacturera, para el caso de los créditos solicitados por las clases trabajadoras a las AAP, así como de formas de producción industrial de la misma (Pradilla, 1987), llevada a cabo, por ejemplo, a apartir del IVU. Lo mismo sucedería para el caso de la vivienda producida en el mercado sin mediación alguna del Estado. Los resultados de esta producción serían valores de uso y de cambio, cuyas propiedades espaciales (Smith, 2006) dirigidas hacia las clases sociales dentro del mercado laboral formal con ingresos medios y altos son cualitativamente superiores a las autoproducidas por las clases populares. Por otra parte, las técnicas asociadas a la producción de vivienda van más allá de las formas y técnicas productivas, incorporando otras técnicas administrativas y burocráticas que surgen de toda la institucionalidad creada, es decir, otras técnicas sociales (Santos, 2000). Con ello se vislumbra el carácter relacional que permite esta desigualdad en la producción de los valores de uso que presenta la producción de las viviendas y las clases o grupos sociales que relizan estos valores de uso.

Complementariamente, durante el periodo también sugieron experiencias de producción de vivieda formal llevadas a cabo por instituciones sin fines de lucro, dirigidas hacia las clases populares, como es el caso de la Fundación Salvadoreña de Desarrollo y Vivienda Mínima (FSDVM, que posteriormente adoptó el acrónimo FUNDASAL), cuyas formas productivas y técnicas también serían superiores a las que intervienen en la generalidad de casos de la autoproducción de vivienda para estas clases.

En conjunto, la producción de viviendas orientadas hacia las clases sociales consideradas como demanda solvente, con ingresos medios o altos, a partir de la intervención del Estado y sus instituciones muestra límites claros. Lungo (1980) registra que entre 1951 y 1973 se produjeron 18,487 viviendas en todo el territorio nacional por parte del IVU; mientras que las viviendas producidas en el AMSS por el IVU, FSV y la FSDVM (estas últimas dirigidas a sectores populares) entre 1972 y 1978 ascendieron a un total de 9,765 viviendas (Lungo, 2000). Estas cifras son pírricas con relación a las 149,178 unidades habitacionales urbanas del AMSS registradas entre los años 1976 y 1977 (Zchaebitz et. al., 1994); lo que 
conduce a inferir que la mayoría de la vivienda producida para las clases sociales de ingresos medios y altos se logró sobre todo desde la puesta en marcha de la FNV y las AAP, así como por las entidades del mercado de vivienda fuera de la intervención estatal. Este proceso, además fue acompañado por otras instituciones estatales que normaron la distribución del espacio a partir de instrumentos de planificación y planes de desarrollo urbano. Tal es el caso del METROPLAN 80, creado en 1968, en el que se establecieron las mayores densidades habitacionales para las zonas de la metrópoli donde se asentaban mayoritariamente las clases populares y las clases trabajadoras formales de menores ingresos, mientras que asignaba a las zonas de habitación de las clases dominantes y de ingresos medios y altos densidades habitacionales muy por debajo de las asignadas a las primeras (Peña, 2001). Así, se normalizó y reguló una distribución nada natural ni preestablecida naturalmente (Martín-Baró, 1986) o neutral de menos espacio para las clases populares y de más espacio para las clases sociales económicamente solventes y dominantes; lo que se corresponde al mismo tiempo con la noción del espacio dominante producido por los grupos dominantes (Harvey, 1994; Lefebvre, 1976), como una clara representación espacial convertida en práctica espacial (Lefebvre, 2013).

Las proporciones de viviendas populares y del resto de viviendas de las clases con ingresos medios y altos en los municipios metropolitanos son concluyentes respecto a la estructura socioespacial originada por la división social del espacio residencial (Duhau, 2013) y sirven para dar cierre a las reflexiones de este artículo. La información proporcionada por Baires (2006) para el año 1974 permite afirmar que los municipios de San Marcos y Soyapango, ubicados entre el sur-oriente y el oriente de la capital, contaban con menos del 25\% de viviendas formales; los municipios de Ciudad Delgado, Cuscatancingo, Mejicanos y Ayutuxtepeque, localizados próximos a la zona central metropolitana, pero al norte y oriente de San Salvador, contaban con menos del 46\% de viviendas formales; mientras que Nueva San Salvador, San Salvador y el municipio de Ilopango, localizado al oriente de la capital, contaban con más del $50 \%$ de viviendas formales, pero sin llegar a representar siquiera dos terceras partes de estas; en tanto Antiguo Cuscatlán contaba con poco menos del $80 \%$ de sus viviendas formales (Tabla 1). 
Carlos Vinicio Coreas-Bonilla

Análisis crítico de la producción de la división social del espacio residencial para el caso del

Área Metropolitana de San Salvador, El Salvador, entre 1950 y 1979. Una interpretación geográfica

Tabla 1. Proporción de viviendas populares y formales por municipio del AMSS en 1974.

\begin{tabular}{lcc}
\hline \multicolumn{1}{c}{ Municipio } & Vividas populares (\%) & Viviendas formales (\%) \\
\hline Nueva San Salvador & 49.2 & 50.8 \\
\hline Antiguo Cuscatlán & 20.6 & 79.4 \\
\hline San Salvador & 44.0 & 56.0 \\
\hline San Marcos & 86.5 & 13.5 \\
\hline Soyapango & 96.1 & 3.9 \\
\hline Ciudad Delgado & 64.1 & 35.9 \\
\hline Cuscatancingo & 72.4 & 27.6 \\
\hline Mejicanos & 62.1 & 37.9 \\
\hline Ayutuxtepeque & 54.9 & 45.1 \\
\hline Ilopango & 36.4 & 63.6 \\
\hline Total & 51.8 & 48.2 \\
\hline
\end{tabular}

Fuente: Elaboración propia con base en Baires (2006)

Los resultados del análisis a la luz del marco teórico y su aplicación al proceso de urbanización, la problemática de vivienda y las políticas estatales de vivienda evidencian la existencia de la división social del espacio residencial a partir de un patrón de localización de las clases dominantes y de las clases de ingresos medios o altos en el vértice sur-poniente de la metrópoli, conformada por los municipios de Nueva San Salvador, Antiguo Cuscatlán y San Salvador. Estos mismos municipios fueron, además, favorecidos por el Estado en la dotación de infraestructuras y servicios diferenciados (Lungo, 1993) respecto al resto de municipios. En los primeros el Estado invirtió "en la adecuación física y el equipamiento básico de la tierra urbana [favoreciendo] claramente a las urbanizaciones de los sectores altos y medios" (Lungo, 2000, p. 75). Adicionalmente, "los centros de oferta cultural y de servicios urbanos se siguen ubicando en el oeste del municipio de San Salvador" (Baires, 2006, p. 51), es decir, a la zona sur-poniente de San Salvador, junto con Antiguo Cuscatlán y Nueva San Salvador; que es la zona hacia donde se han ido desplazando históricamente las clases dominantes y sectores medios (Lungo y Baires, 2001). La práctica espacial que origina la producción de la división social del espacio 
residencial ha sido la concreción material de las representaciones espaciales de la política de vivienda y de la planificación urbana, en conjunto con los sectores privados capitalistas que han diseñado las viviendas y el lugar de localización para las clases dominantes y las clases de ingresos medios; y, por lo tanto, el no lugar para las clases populares.

Relacionalmente sucede lo opuesto respecto a las clases populares, con excepción de Ilopango. La localización de viviendas populares en sus diferentes tipologías se concentra en mayor proporción en municipios que se alejan del centro de la capital, formando un arco que corre desde el norte de San Salvador hacia el sur y oriente. Según Lungo (1996), esta periferización sería en parte el resultado del poblamiento de las clases populares por medio de colonias ilegales en las tierras suburbanas que contribuyeron al crecimiento metropolitano; pero que de acuerdo con FUNDASAL (1997b) no contaban con redes de infraestructura o servicios comunitarios, donde la vivienda fue autoproducida. A las colonias ilegales se agregan los tugurios que se concentran en su mayoría en la capital (71.3\%), seguidos por aquellos localizados en Ciudad Delgado, Mejicanos y Soyapango (20.3\% en conjunto) (USAID, 1985), siguiendo el patrón del arco periférico señalado previamente. Por su parte, los campamentos y mesones tendrían mayor dispersión respecto al centro de la capital, como se mostró en el Mapa 1.

Como se ha sostenido, la producción social de la división social del espacio residencial del AMSS entre 1950 y 1979 se materializa por medio de las prácticas espaciales a las que se refiere Lefebvre (2013), en el marco de la reproducción de las relaciones sociales de producción en el espacio geográfico como totalidad y también en el espacio urbano de la metrópoli, originando patrones identificables respecto a la localización de las diversas clases sociales y sus respectivas viviendas (Smith, 2006). Las causas últimas que subyacen a este proceso de producción socioespacial se encuentran en las relaciones sociales de clase y su posición dentro de la estructura económica en el marco del modo de producción capitalista (Marx 1995). Por esta misma razón, las formas productivas de las viviendas y las técnicas que intervienen para su construcción también se corresponden con las distintas clases sociales (Pradilla, 1987; Santos, 1990, 2000); todo ello, considerando el carácter relacional entre estas (Feito, 2007; Wright, 2010; Crompton, 2013 y Pérez Sáinz, 2014), así como de la desigualdad 
socioespacial heterogéna y múltipe producida en la que se reflejan los procesos sociales dirigidos por los poderes domiantes del capital y del Estado (Harvey, 1977, 1994), que son al mismo tiempo los que definen la cuota que le responde a cada individuo en función de su clase por medio de las leyes de la distribución social de lo producido (Pradilla, 1984).

\section{Referencias}

Ariza, M. y Solís, P. (2009). Dinámica socioeconómica y segregación espacial en tres áreas metropolitanas de México, 1990 y 2000. Estudios Sociológicos, 27(79), 171-209. Recuperado de http://www.jstor. org/stable/25614137

Baires, S. (2006). División social del espacio urbano y emergencia de los barrios cerrados en el Área Metropolitana de San Salvador. A. En Séguin y P. Negrón (Ed.), La segregación socio-espacial urbana: una mirada sobre Puebla, Puerto España, San José y San Salvador. (47-84). San José, Costa Rica: FLACSO Costa Rica. Recuperado de: http://enlaceacademico.ucr.ac.cr/node/2467

Barba, J. (1998). Gobernar desde las ciudades. La expansión metropolitana de San Salvador. En V. Herrera (Ed.), Gobernabilidad urbana en Centroammérica. (65-93). San José, Costa Rica: FLACSO Costa Rica. Recuperado de http://enlaceacademico.ucr.ac.cr/node/2988 Castells, M. (1976). La cuestión urbana. (2ª ed.) México, D.F.: Siglo XXI. Coreas, C. (2014). Clases sociales y pobreza en El Salvador, 2000-2012. (Tesis de Maestría en Población y Desarrollo). FLACSO, México. Recuperado de http://flacso.repositorioinstitucional.mx/jspui/ handle/1026/53

Crompton, R. (2013). Clase y estratificación: una introducción a los debates actuales. Madrid, España: Editorial Tecnos.

Duhau, E. (2003). División social del espacio metropolitano y movilidad residencial. Papeles de Población, 9 (36), 161-210. Recuperado de http://www.redalyc.org/articulo.oa?id=11203608

Duhau, E. (2013). La división social del espacio metropolitano: Una propuesta de análisis. Nueva Sociedad, (243), 79-91. Recuperado de http://nuso.org/media/articles/downloads/3917_1.pdf

Feito, R. (1997). Estructura social contemporánea. Las clases sociales en los países industrializados. Madrid, España. Siglo XXI España. 
Ferrufino, C. (2013). La provisión de suelo y vivienda accesible en El Salvador. Documento de trabajo del Lincoln Institute of Land Policy. Recuperado de https://www.lincolninst.edu/sites/default/files/pubfiles/lopez-wp14cf1sp-full_0.pdf

Filgueria, C. (2001). La actualidad de viejas temáticas: sobre los estudios de clase, estratificación y movilidad social en América Latina. CEPAL. Recuperado de https://www.cepal.org/es/publicaciones/6008-la-actualidad-viejas-tematicas-estudios-clase-estratificacion-movilidad-social

Fontg, M., Barrientos, M. y Figueroa, A. (1991). Liberalización financiera y sistema de ahorro y préstamo en El Salvador. San Salvador, El Salvador. Realidad, (21), 299-325. Recuperado de http://www.uca.edu. sv/revistarealidad/?pag $=$ revista\&idrevista $=108$

Fundación Salvadoreña de Desarrollo y Vivienda Mínima (FUNDASAL) (1993a). Una Lectura Histórica de las Políticas de Vivienda de El Salvador (Parte II). Carta Urbana, (18), 1-12.

Fundación Salvadoreña de Desarrollo y Vivienda Mínima (FUNDASAL) (1993b). Políticas Estatales de Vivienda y Programas habitacionales en El Salvador. Carta Urbana, (20), 1-12.

Fundación Salvadoreña de Desarrollo y Vivienda Mínima (FUNDASAL) (1994). La evolución de Asentamientos Populares en el Área Metropolitana de San Salvador. Carta Urbana, (27), 1-17.

Fundación Salvadoreña de Desarrollo y Vivienda Mínima (FUNDASAL) (1997a). Los tugurios en El Salvador. Carta Urbana, (52), 1-12.

Fundación Salvadoreña de Desarrollo y Vivienda Mínima (FUNDASAL) (1997b). El mercado de lotificaciones suburbanas en El Salvador. Carta Urbana, (53), 1-12.

Fundación Salvadoreña de Desarrollo y Vivienda Mínima (FUNDASAL) (1999). Intervenciones públicas y privadas en zonas tugurizadas. Carta Urbana, (71), 1-16.

Goldthorpe, J. (2010). De la sociología. Números, narrativas e integración de la investigación y teoría. (Vol. 1), Madrid, España. CIS.

Griffith, K. y González, L. (1999). Notas sobre la "autonomía" del Estado, El caso de El Salvador. Estudios Centroamericanos 612(LIV), 893-910.

Hall, T. (2001). Urban Geography. (2 ${ }^{\mathrm{a}}$ ed.). Routledge. 
Carlos Vinicio Coreas-Bonilla

Análisis crítico de la producción de la división social del espacio residencial para el caso del

Área Metropolitana de San Salvador, El Salvador, entre 1950 y 1979. Una interpretación geográfica

Harth, A. (2013). Perfil del Sector Vivienda de El Salvador. Nairobi, Kenya. ONU-Habitat. Recuperado de https://unhabitat.org/books/ perfil-del-sector-vivienda-de-el-salvador/

Harvey, D. (1977). Urbanismo y desigualdad social. Madrid, España. Siglo XXI

Harvey, D. (1994). La construcción social del espacio y del tiempo: una teoría relacional. Geographical Review of Japan, 67(2), 126-135.

Herrera,A.y Martín-Baró,I.(1978). La ley y orden en la vida del mesón. Estudios Centroamericanos 33(360), 803-828 Recuperado de http://www.uca.edu. sv/coleccion-digital-IMB/articulo/ley-y-orden-en-la-vida-del-meson/

Lefebvre, H. (2013). La produccion del espacio. Madrid, España. Capitán Swing.

Lefebvre, H. (1976). Espacio y política. Barcelona, España. Edicions 62. López Bernal, G. (2015). Las claves de la historia de El Salvador. El Salvador. Historia contemporánea, 1808-2010. (27-51). López Berlan (Ed.), San Salvador, El Salvador: Fundación MAPFREC y Editorial Universitaria de El Salvador.

López Pérez, C. (1986). Industrialización y urbanización en El Salvador, 1969-1979. San Salvador, El Salvador. UCA Editores.

Lungo, M (1980). El Salvador. 1932-1978. La crisis del Estado y el papel de las "Políticas Sociales". (Acerca de las funciones que cumplen las politicas de vivienda). San Salvador, El Salvador: Facultad de Ciencias y Humanidades, Universidad de El Salvador.

Lungo, M. (1993). La urbanización del Área Metropolitana de San Salvador: tendencias a partir de 1970 e ideas preliminares para un desarrollo urbano alternativo. San Salvador, El Salvador. Avances, (1), 1-31. Lungo, M. (1996). La gestión de la tierra urbana en El Salvador. Prisma, (20), 1-11.

Lungo, M. (2000). La tierra urbana. San Salvador, El Salvador. UCA Editores.

Lungo, M. y Baires, S. (2001). Socio-spatial segregation and urban land regulation in Latin American cities. International Seminar on Segregation in the City. Cambridge, MA, USA. Recuperado de http://citeseerx.ist.psu.edu/viewdoc/summary?doi=10.1.1.200.8130\&rank=1

Martín-Baró, I. (1986). El hacinamiento residencial: ideologización y verdad de un problema real. Revista de Psicología de El Salvador, 
9(35), 23-51. Recuperado de http://www.uca.edu.sv/coleccion-digital-IMB/articulo/hacinamiento-residencial-ideologizacion-y-verdad-de-un-problema-real/

Marx, K. (1995) El Capital. Crítica a la economía política (Tomo III). México, D.F., México. Fondo de Cultura Económica

Montes, S. (1979). Estudio sobre estratificación social en El Salvador. San Salvador, E1 Salvador. UCA Editores.

Montoya, A. (1995). Informalidad urbana y nueva economía popular. San Salvador, El Salvador. UCA Editores.

Peña, R. (2001). Normas y regulaciones urbanísticas y su papel en la segregación socio-espacial en el Área Metropolitana de San Salvador. (Tesis de maestría). FLACSO Costa Rica.

Pérez, J. (2014). Mercados y Bárbaros. La persistencia de las desigualdades de excedente en América Latina. San José, Costa Rica. FLACSO Costa Rica. Recuperado de http://enlaceacademico.ucr.ac.cr/ node $/ 2150$

Pradilla, E. (1984). Contribución a la crítica de la "teoría urbana". Del "espacio" a la "crisis urbana”. México, D.F., México. UAM Xochimilco. Recuperado de http://www.emiliopradillacobos.com/Libros.htm

Pradilla, E. (1987). Capital, Estado y vivienda en América Latina. México, D.F., México. Fontanamara S.A. Recuperado de http://www.emiliopradillacobos.com/Libros.htm

Romero, M. y Vides, M. (2006). Análisis de la inversión en el sector de la vivienda popular en El Salvador. Periodo 1995-2005. (Tesis de Licenciatura en Economía). Universidad Centroamericana "José Simeón Cañas" UCA. San Salvador, El Salvador. Recuperado de http://www.uca.edu.sv/deptos/economia/?art=111

Rubalcava, R. y Schteingart, M. (2012). Ciudades Divididas. Desigualdad y segregación social en México. México, D.F., México. El Colegio de México.

Salgado, M. (2016). Estado y desarrollo en Centroamérica: trayectorias recientes y alternativas posibles. (Tesis doctoral en Economía). UNAM, México. (Inédito)

Santos, M. (1990). Por una geografía nueva. Madrid, España. EspasaCalpe, S.A. 
Carlos Vinicio Coreas-Bonilla

Análisis crítico de la producción de la división social del espacio residencial para el caso del

Área Metropolitana de San Salvador, El Salvador, entre 1950 y 1979. Una interpretación geográfica

Santos, M. (2000). La naturaleza del espacio. Técnica y tiempo, razón y emoción. Barcelona, España. Ariel, S.A

Saraví, G. (2008). Mundos aislados: segregación urbana y desigualdad en la ciudad de México. EURE, XXXIV (103), 93-110. Recuperado de http://www.eure.cl/index.php/eure/article/view/1367

Segura, R. (2014). El espacio urbano y la (re)producción de desigualdades sociales. Desacoples entre distribución del ingreso y patrones de urbanización en ciudades latinoamericanas. Working Paper Series 65. Berlín, Alemania. Recuperado de http://www.desigualdades.net/ Working_Papers/index.html.

Smith, N. (2006). La producción de la naturaleza. La producción del espacio. México, D.F., México. UNAM. Facultad de Filosofía y Letras, Sistema de Universidad Abierta.

Soja, E. (2008). Postmetrópolis. Estudios críticos sobre las ciudades y las regiones. Madrid, España. Traficantes de Sueños.

Solís, P. y Benza, G. (2013). Clases sociales, pobreza y desigualdad en los años de alternancia presidencial. México, D.F., México. (Inédito)

United States Agency for International Development (1985). El Salvador. Shelter Sector Assessment. Washington, DC., USA. USAID.

Velásquez, C. (2012). La evolución del Estado en El Salvador durante el siglo XX: el giro neoliberal y las continuidades clasistas. El Estado en América Latina: continuidades y rupturas. (227-252). Santiago, Chile. CLACSO - Editorial Universidad ARCIS. Recuperado de http://biblioteca.clacso.edu.ar/clacso/gt/20121127121700/ElEstadoenAmericaLatina.pdf

Walter, K. y Williams, P. (2011). El papel político del Ejército salvadoreño. Sader, E. (Ed.) El Salvador: Historia mínima. (71-82). San Salvador, El Salvador. Secretaría de Cultura de la Presidencia de la República. Wright, E. (2010). Preguntas a la desigualdad. Ensayos sobre análisis de clase, socialismo y marxismo. Bogotá, Colombia. Editorial Universidad del Rosario.

Zschaebitz, U., Serarols, J. y Portillo, R. (1994). Estadísticas básicas de población y vivienda del Área Metropolitana de San Salvador, 1950/1971-1993. Contexto del estudio de los asentamientos populares urbanos. Documentos de Estudio FUNDASAL 14. Recuperado de http://repo.fundasal.org.sv/204/ 